\title{
Role of Platelet-Mediated Cytoadherence and Chemokines Release in Severe Malaria
}

\author{
Kalyan Srivastava ${ }^{1 *}$, Kamna Srivastava ${ }^{2}$ \\ ${ }^{1}$ New York Blood Center, New York, USA \\ ${ }^{2}$ Dr. Ambedkar Center for Biomedical Research, University of Delhi, India
}

Received: January 09, 2015; Accepted: May 30, 2015; Published: August 30, 2015

*Corresponding author: Kalyan Srivastava, New York Blood Center, New York, USA, Tel: +805630260, E-Mail: kalsriv@gmail.com

\begin{abstract}
Absract
Platelets play an important role in the pathogenesis of malaria infection. Platelets' action is mostly considered to be mediated by their immune effects. Platelets release thrombo-inflammatory agents such as chemokines, cytokines and coagulatory agents that may contribute to the pathogenesis of the disease. They can also participate in cytoadherence to the micro vessels of organs such as brain, lungs and spleen. Both immune response and cytoadherence can act in tandem to cause blood organ (e.g. brain) barrier breakdown causing damage. Circulating infected red blood cells interact with platelets in a receptor-mediated process that results in platelet activation and chemokines and inflammatory cytokines release. In this review, we intend to provide evidence that indicate the platelets' role in cytoadherence and immune response. We have mostly used the example of cerebral malaria; however, a similar mechanism involving platelets might contribute, to understand the pathogenesis in other cases of severe malaria.
\end{abstract}

Keywords: Cytoadherence; Chemokines; Cerebral malaria; Severe malaria; CXCL4; CXCR3; P. falciparum

\section{Introduction}

Malaria is a mosquito-borne infection caused by Apicomplexan parasites of the genus Plasmodium. These parasites use two hosts and various tissues for their multiplication and survival: the mosquito gut and mammalian erythrocyte and hepatocytes. Every year, malaria affects more than 500 million people, both inhabitants and visitors, of the endemic regions. The disease causes death, disability and economical damage [1] in these zones. Malaria infection results in a variety of clinical syndromes such as Severe Malarial Anemia (SMA), Cerebral Malaria (CM), Placental Malaria (PM) and Malaria Related Acute Lung Injury (MALI). SMA is accompanied by symptoms such as fever, anemia and acidosis. CM is a neurological manifestation of malaria that might result in coma, ataxia and permanent neurological damage. PM affects pregnant women and the fetus causing abortion, anemia and maternal death. MALI is a pulmonary manifestation resulting in damage to the lungs. Each of these conditions is potentially fatal and results from the host-pathogen interaction. The role of platelets in the host's immune response and malaria pathogenesis is widely recognized. In this context, platelets mediated damage encompasses each class of malaria syndromes i.e. SMA, CM, PM and MALI. It has been suggested that platelets play a dual role [1] participating in parasite clearance by activation of the hosts' innate immune system and releasing platelet-specific chemokines such as CXCL4/PF4 and RANTES and [2] by activating an exaggerated innate immune response. The latter is usually a consequence of specific immune responses leading to clear the parasite from peripheral blood and causing tissue and microvessel damage in the host.

Platelets are anucleated and terminally differentiated cells derived from megakaryocytic, the thrombogenic progenitor cells. They serve as a first line of defense against loss of vascular integrity. They also participate in atherosclerosis and thrombosis in pathological conditions. In recent years, platelets' role beyond hemostasis and thrombosis has been identified. Sophisticated proteomics studies have been able to estimate $>500$ protein in platelets [2]. These proteins are either inherited from megakaryocytic, or freshly translated from the RNA pool. They have been categorized based on their involvement in functions such as coagulation thrombo-immunological, endocrine, apoptosis, tumor and various signaling events. Among these functions, the immune response has recently gained immense interest. These immune-related proteins are packed in various cellular compartments especially the granules i.e. alpha, dense and lysosomal [3]. We believe that platelets can participate in cytoadherence as well as the immune response which are the hypothesis of this article.

\section{Mouse model of Experimental Cerebral Malaria(ECM)}

The Experimental Cerebral Malaria-Model (ECM) is an extensively exploited method for studying cerebral malaria in vivo [4-6]. In general, the model involves intraperitoneal injection of $10^{6}$ P. berghei ANKA parasites into the C57Bl6 mice, which shows cerebral manifestation by the $5^{\text {th }}$ day and mortality by the $6^{\text {th }}$ day. This is due to its high histological, immunological and neurological similarity with the human equivalent. The availability of knock-out mice has accelerated the study on the role of chemokines in ECM. Other groups have used P. yoelii 17XL mice injection in C57bl6 mice as ECM. However, a combination of P. berghei ANKA and C57bl6 is being widely used for ECM studies. 


\section{Platelet cytoadherence and cerebral malaria}

Cytoadherence is a crucial step for the pathogenesis initiated by $P$. falciparum in humans and the equivalent parasite in experimental animal models. P. falciparum parasites export their erythrocyte membrane protein 1 (PfEMP1), encoded by variable genes (var genes), to the surface of infected erythrocytes (iRBC). This enables them to adhere to the microvasculature and assist in evasion from immune clearance by the spleen. Experimental blocking of these cytoadherence results in increased mature iRBC in the circulation, supporting this hypothesis. There are approximately 60 var genes in each parasite genome. However, only one is actively transcribed at a time. The remaining genes are kept silent through a molecular process called "mutually exclusive expression" [7]. It has been suggested that cytoadherence is a critical step for the progression of CM. Cytoadherence leads to the activation of endothelial cells in brain's microvessels and promotes endothelial cell rigidity and apoptosis. iRBCs adhere to the endothelial cells of brain's microvessels and initiate neuroinflammatory processes and vascular inflammation. This recruitment of $\mathrm{IRBC}$ to the endothelium can be direct or mediated by platelets. iRBC accumulation in brain microvessel results in a threefold increase in Blood Brain Barrier (BBB) permeability, which causes leakage of the proteins from serum into the central nervous system. The presence of these foreign proteins results in activation of the microglia, and further release of proinflammatory cytokines causing damage to astrocytes, pericytes and other glial cells.

There is strong evidence that sequestration, a type of cytoadherence, of infected erythrocytes iRBC in brain microvessel plays a key role in CM pathogenesis [8-10]. Studies suggest that the most effective anti-parasite therapy may not be able to guarantee $100 \%$ recovery in case of severe malaria. Indeed, sequestered, parasites rapidly return to circulation once the therapy is completed. An important consequence of the cytoadherence phenomenon is the injury of microvessels and consequently, organ damage. Therefore, it is important to administer therapy combining the blocking of cytoadherence with antimalarial drugs for complete recovery. It must also be noted that switching to a different class of var gene can also contribute to immune evasion by the parasites. Therefore, studies involving the role of platelet in cytoadherence are necessary from the perspective of variant transcription of Pf EMP.

Since mice expressing var gene binding proteins on RBC are still under development, most researchers use in vitro studies [11]. A study on children with CM and Uncomplicated Malaria (UM) from South Benin, Africa [12] investigated the cytoadherence phenotypes of iRBC regarding CD36, ICAM-1, and CSPG with numerous var gene transcripts. The study helped in determining the cytoadherence during CM in relationship to a specific transcription profile of Pf EMP-1 variants. In addition, the authors showed increased binding of iRBC to CD36 in CM patients compared to UM. The study further demonstrated that CM isolates highly transcribed var gene groups A, B, var2csa, var3, DC8 and DC13 compared to UM parasites and that group $\mathrm{B}$ protein binding to $\mathrm{CD} 36$ is predominantly responsible for
CM progression. However, it fell short in explaining the specific domain from group B protein that may result in binding. To be therapeutically relevant, the domain of protein involved in binding must be identified. This binding domain could further be used as a target for the development of antibody or inhibitor. Also, this study failed to address the role of platelets in CM pathogenesis. The possibility of a relationship between a parasite's switch to a different class of var gene enabling adhesion to the vascular bed and platelet's presence in spatial-temporal manner, should be considered when exploring underlying mechanism in $\mathrm{CM}$ pathogenesis. Therefore, an elaborate study is needed to understand the role of platelet-mediated cytoadherence in CM using similar expression cassettes. Numerous laboratories have attempted to develop var gene transcript expressing mice for the study of the mouse model of cytoadherence [12]. This would reveal the dependence of each category of malaria pathogenesis with different var gene products.

We have demonstrated a role of CD36 in platelet activation in case of malaria infection [4]. Although CD36 is an important platelet receptor in the process, the involvement of platelet surface ICAM-1 cannot be ruled out. Another cell surface protein expressed both on endothelial cells and platelets is the Von WilleBrand Factor (vWf). Expression of this factor is observed high in the serum of patients with malaria [5]. The role of vWf in CM cytoadherence is less studied than in other conditions. In the diseases such as atherosclerosis and thrombosis, vWf is considered as a diagnosis marker and is often the target of therapy [13,14]. Similarly, therapies in autoimmune diseases have also suggested the targeting of CD36 and ICAM-1 in various conditions [15]. Another theory proposes a combination of host-mediated cytoadherence and proinflammatory cytokines, preferably released by platelets, working in tandem to cause cerebrovascular damage [16]. Therefore, the study involving chemokines in combination with cytoadherence is important for complete understanding of the pathogenesis of the condition [17].

\section{Platelet -specific cytokines and cerebral malaria}

Another aspect of the infection, equally important as cytoadherence, is the expression of cytokines during the acute phase of the disease. All this work has demonstrated the role of a platelet-specific chemokines, CXCL4 (Platelet Factor 4 or PF4), in CM using an ECM model [4]. CM is a cerebral manifestation of malarial infection and mostly afflicts the children under the age of five in the endemic zone. Pathogenesis of CM includes the brain's micro-capillary obstruction, inflammation and BBB breakdown. We have demonstrated that the CXCL4 participates in the progression of CM. Also, CXCL4-mediated cerebral damage is accompanied by T-Cell and monocyte activation plus consequent BBB breakdown [18-21]. Both platelets and monocytes can release the cytokines upon the onset of $\mathrm{CM}$. We also demonstrated the involvement of platelet surface CD36, associated with cytoadherence and activation of platelets and responsible for the release of cytokines such as CXCL4. We have also performed studies showing that CXCL4-/- mice have reduced mortality from CM [4]. Damage caused by CXCL4 progresses through T-Cell and 
monocyte activation and migration resulting into BBB breakdown [19-21], which in turn also results in increased permeability of cerebral micro-capillaries. Other groups have also demonstrated that T-Cell activation is an important step in CM progression [22]. Another set of studies, conducted by us included the treatment of ECM mice with interferon beta [23] and Resveratrol (in press). These rescue processes progress through the suppression of T-Cell migration as shown in these experiments. Therefore, it is clear that effects of platelet secreted chemokines are mediated by the activation of T-cells, monocyte and macrophage cells. In ECM studies, both CD8+ T-cells and CD4+ T-cells were found to be activated by CXCL4 overexpression. In addition, our work on knockout mice has demonstrated that CXCR3, a G Protein Couple Receptor (GPCR), is activated and participated in pathogenesis [4]. The receptor CXCR3 is highly expressed on T-cells and has been widely studied in other diseases. The CXCR3-/- mice are completely rescued from death in ECM model. Our study has clearly indicated that the axis of "CXCL4-CXCR3 -T-cell activation" is critical in the CM pathogenesis.

Besides its role on pathology, CXCL4 also participates in parasite clearance in early stages of the infection [24]. This is in the harmony with the recently discovered fact that platelets participate in early protective phases during malaria, which suggests that reduction in the count of platelets or the CXCL4 neutralization cannot be considered as a therapeutic measure. There is a possibility that, from an evolution point of view, baseline serum levels of CXCL4 might have granted a survival advantage to the infected host. In addition, the receptor CXCR3 is vital for various cellular functions, therefore, blocking of these receptors can be potentially harmful. This undermines the CXCL4/CXCR3 interaction blocking as a target for CM treatment. However, CXCL4 can also act via heteromerization with other chemokines to induce pathogenesis, for instance- CXCL4/CCL5 and CXCL4/IL8 heteromerization [25]. Moreover, the heteromeric form of CXCL4 may also bind to receptors other than CXCR3, for the activation of T-cells. Blocking these heteromerization may require further studies and can be a valuable therapeutic target as it has been shown in the case of aortic aneurysm [26].

In addition to CXCL4, numerous other platelet-specific chemokines and inflammatory cytokines have been identified. These chemokines are either produced by platelets or taken up by them at a different site and delivered at the affected tissue. These chemokines are released upon platelet activation and participate in a variety of inflammatory processes. Few examples of important platelets secreted chemokines include CCL5, CXCL5, CXCL7, CXCL12, CXCL16, MIF and plasminogen inhibitor [27]. These chemokines play an important role in vascular inflammation. While many of them have clear involvement in T-cell activation, not much has been explored in relation to the CM. It is feasible that chemokines act alone or in combination with other chemokines to activate T-cells. Simultaneously, they might activate other pathogenic immune cells including monocytes, macrophage and leukocytes. In humans, T-cells express different receptors which respond to platelet-specific chemokines; these include CCR1, CCR5, CXCR1, CXCR2, CXCR4, and CXCR6. The chemokines CXCL7, highly expressed during platelet activation, is associated with malaria infection and binds to CXCR1 and CXCR2 receptors. The chemokines, CCL5, binds to CCR1 and CCR5 and can activate T-cells. Platelets also express CXCL12 which binds to the T-cells' CXCR4 receptor. The chemokines CXCL16, expressed in platelets is relatively less studied. This chemokine exclusively binds to CXCR6 receptors and it has a vital role in T-cell activation and migration. The chemokines CXCL5 and MIF may also have a role in T-cell migration and need to be studied in the context of CM pathogenesis. Studies have demonstrated that serum values of these chemokines are either elevated or reduced upon infection. For instance, CXC12 levels are reduced in relation with malaria pathogenesis. In addition, these chemokines can also act via heteromerization as explained before. However, the role of each of these chemokines and their receptors requires investigation in cases of cerebral malaria using ECM models [28].

\section{Platelets and MALI and PM}

One less studied form of pathogenesis arising during $P$. falciparum infection is known as malaria acute lung injury (MALI) [29]. It has been observed in non-juvenile patients with malaria and involves pulmonary damage. There is strong evidence suggesting that platelets play an important role in nonmalaria Acute Lung Injury (ALI). ALI is highly correlated with neutrophils activation in diseases which may be accompanied by platelets activation and release of CXCL4. The human lungs possess megakaryocytes that can actively produce highly reactive platelets. During ALI, platelets have been found to directly interact with neutrophils by engaging numerous cell surfaces receptors such as Glycoprotein Ib and Glycoprotein IIb Beta III [30]. Also, CXCL4 has been found to initiate various processes that can activate neutrophils and promote alveolar infiltration. The involvement of this platelet-leukocyte interaction in malaria pathology requires experimental confirmation. An interesting starting point for these studies could be the exploration of the platelets activation and interaction conducting to cytoadherence and promoting MALI [31]. Several mice models have been developed to study the chemokines' role in MALI. These include infecting mice with $P$. berghei to reach high parasite as until respiratory distress is developed. These studies would help to understand not only the platelets but also the T-cell's role in MALI. In recent years, the role of T-cells in the pathogenesis of non-malaria related ALI has also been explained [32,33]. It is possible that the activation of T-cells can be mediated by expression of platelet-related chemokines both in ALI and MALI. Therefore, it is imperative to conduct studies highlighting the role of platelet-specific chemokines in the progression of MALI with respect to T-cell activation. Similarly, placental malaria (PM) has also been associated with the platelet mediated cytoadherence and cytokines release [34]. Since PM continues to be a serious condition for mothers, a systematic study is expected to further contribute to outline the role of platelets. Mice expressing human var gene transcripts would help in further understanding of this process.

\section{Conclusion and Future Direction}

Pathogenesis in severe malaria is highly correlated with 


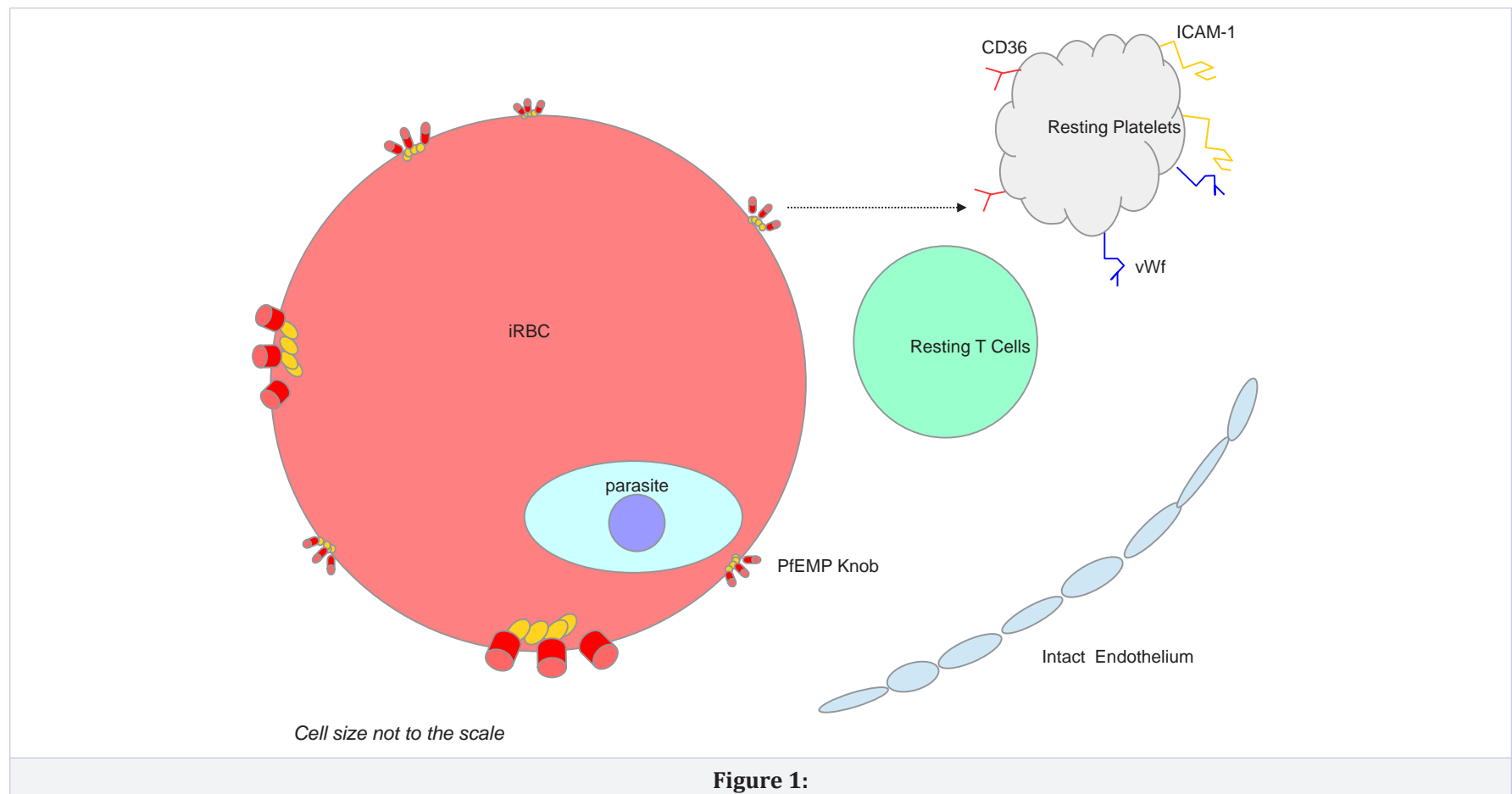

Figure 1:

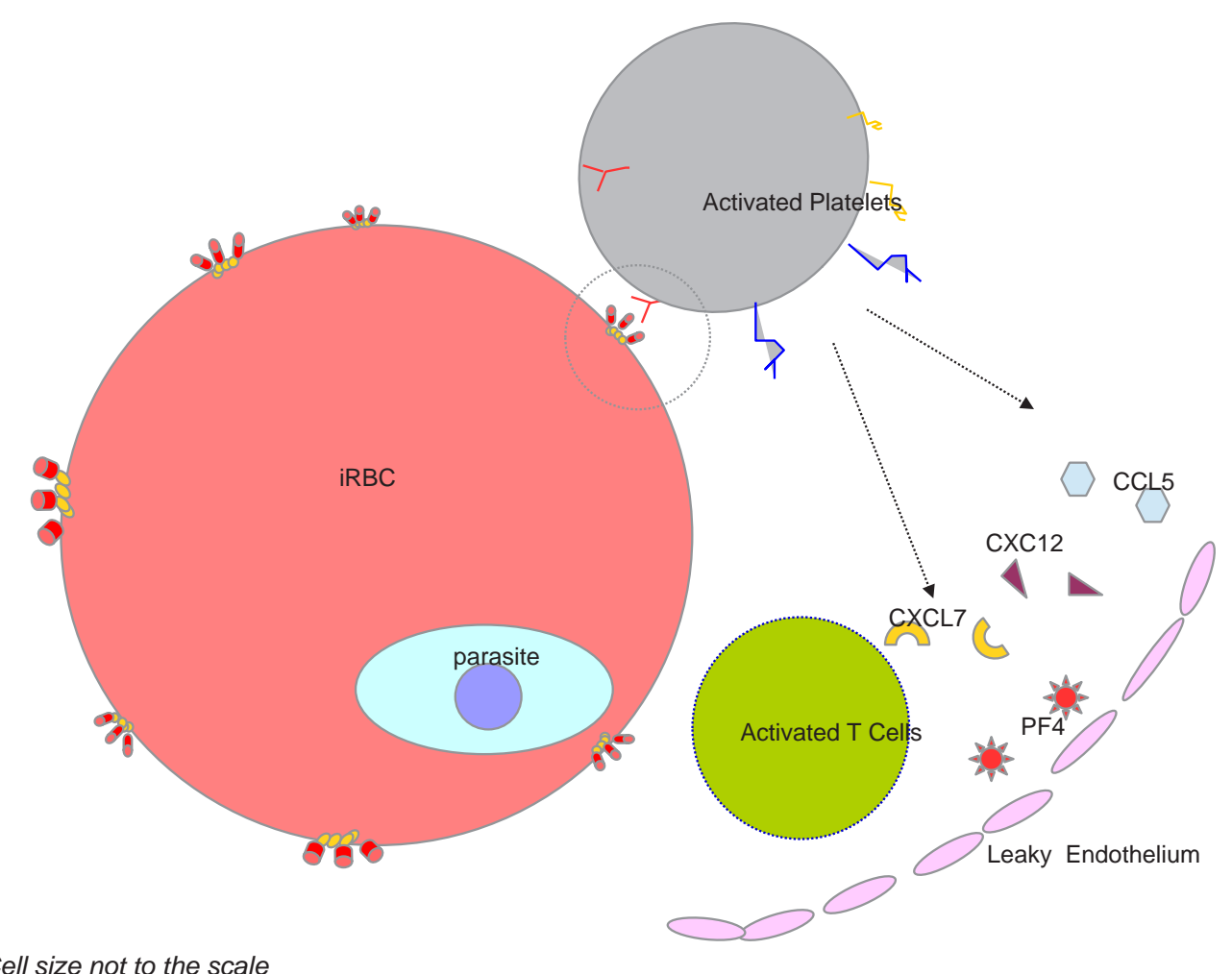

Figure 2: iRBC expresses numerous parasite protein encoded by parasite survival gene and multiplication. One such protein is Pf EMP that is expressed on the surface of RBCs. This protein participates in cytoadherence and attaches itself to the endothelial cells of the microvessels. This interaction is mediated by surface protein such as CD36, vWF or ICAM. iRBC may also interact with other cells such as platelets and participate in the sequestration process resulting into the microvessel obstruction and release of chemokines. Cytokine release and activation of immunological cells such as monocytes and T-cells result into microvessel inflammation. Both inflammation and cytoadherence result in the breaking of blood -brain barrier. Image is an approximation and does not represent the actual size of the cells. 
platelets activation. Both platelet-mediated cytoadherence as well as chemokines release, appear to be equally responsible for pathogenesis. A unified hypothesis combining both plateletmediated cytoadherence and chemokines release is being accepted in CM and can also be acceptable for other types of severe manifestation of malaria. As shown in figures 1,2 onset of malaria can activate the platelet due to interaction with iRBC. Activated platelets can adhere to endothelial cells causing obstruction and sequestration. In addition, this might cause a local and overall increase in inflammatory cytokines and chemokines resulting vascular inflammation and damage. However, as discussed in this review, most of the observations are preliminary and require further exploration before their application in therapeutic strategies. Therefore, further investigation is required to identify downstream of platelet activation that can be use as a target for therapy. In future, it would be beneficial to perform studies to understand the role of platelet-specific cytoadherence, chemokine release and T-cell activation in the pathogenesis of CM, MALI and PM. Commonly expressed platelet specific chemokines such as CXCL7 and CCL5 (and their receptor's) may have a role in T-cell activation during CM. ECM studies engaging global and conditional knockout mice with unexplored chemokines and their receptors might give a valuable information that can help in understanding the pathogenic process. It might also prove valuable to explore the miRNA expression by platelets participating in immune responses. Finally, the involvement of hematopoietic progenitor cells, that may get affected by platelet-related chemokines during severe malaria and platelet interactions with hematological stem cells are other important aspects worthwhile to explore [35].

\section{References}

1. Investigators team (2014): Global, regional and national incidence and mortality for HIV, tuberculosis, and malaria during 19902013: a systematic analysis for the Global Burden of Disease Study 2013. Lancet. 2014; 384(9947):1005-1070. doi: 10.1016/S01406736(14)60844-8.

2. Burkhart JM, Gambaryan S, Watson SP, Jurk K, Walter U, Sickmann $\mathrm{A}$, et al. What can proteomics tell us about platelets? Circ Res.2014;114(7):1204-1219.doi:10.1161/CIRCRESAHA.114.301598.

3. Morrell CN, Aggrey AA, Chapman LM, Modjeski KL. Emerging roles for plateletsasimmuneandinflammatory cells. Blood.2014;123(18):27592767. doi: 10.1182/blood-2013-11-462432.

4. Srivastava K, Cockburn IA, Swaim A, Thompson LE, Tripathi A, Fletcher CA, et al. Platelet factor 4 mediates inflammation in experimental cerebral malaria. Cell Host Microbe. 2008;4(2):179-187. doi: 10.1016/j.chom.2008.07.003.

5. Hollestelle MJ, Donkor C, Mantey EA, Chakravorty SJ, Craig A, Akoto $\mathrm{AO}$, et al. von Willebrand factor propeptide in malaria: evidence of acute endothelial cell activation. Br J Haematol. 2006; 133(5): 562569.

6. Lou J, Lucas R, Grau GE. Pathogenesis of Cerebral Malaria: Recent Experimental Data and Possible Applications for Humans. Clin Microbiol Rev. 2001; 14(4): 810-820, table of contents.

7. Deitsch KW, Calderwood MS, Wellems TE. Malaria: Cooperative silencing elements in var genes. Nature. 2001; 412(6850):875-876.
8. MacPherson GG, Warrell MJ, White NJ, Looareesuwan S, Warrell DA. Human cerebral malaria. A quantitative ultrastructural analysis of parasitized erythrocyte sequestration. Am J Pathol. 1985;119(3):385401.

9. Pongponratn E, Turner GD, Day NP, Phu NH, Simpson JA, Stepniewska $\mathrm{K}$, et al. An ultrastructural study of the brain in fatal Plasmodium falciparum malaria. Am J Trop Med Hyg. 2003;69(4):345-359.

10. Su XZ, Heatwole VM, Wertheimer SP, Guinet F, Herrfeldt JA, Peterson DS, et al. The large diverse gene family var encodes proteins involved in cytoadherence and antigenic variation of Plasmodium falciparuminfected erythrocytes. Cell. 1995;82(1):89-100.

11. Vaughan AM, Kappe SH, Ploss A, Mikolajczak SA. Development of humanized mouse models to study human malaria parasite infection. Future Microbiol. 2012;7(5):657-665. doi: 10.2217/fmb.12.27.

12. Almelli T, Ndam NT, Ezimegnon S, Alao MJ, Ahouansou C, Sagbo G, et al. Cytoadherence phenotype of Plasmodium falciparum-infected erythrocytes is associated with specific pfemp-1 expression in parasites from children with cerebral malaria. Malar J. 2014;13: 333. doi: 10.1186/1475-2875-13-333.

13. Bickel C, Rupprecht HJ, Blankenberg S, Espiniola-Klein C, Schlitt A, Rippin G, et al. Relation of markers of inflammation (C-reactive protein, fibrinogen, von Willebrand factor, and leukocyte count) and statin therapy to long-term mortality in patients with angiographically proven coronary artery disease. Am J Cardiol. 2002;89(8):901-908.

14. De Meyer SF, Stoll G, Wagner DD, Kleinschnitz C. von Willebrand factor: an emerging target in stroke therapy. Stroke. 2012;43(2):599606. doi: 10.1161/STROKEAHA.111.628867. Epub 2011 Dec 15.

15. Yusuf-Makagiansar H, Anderson ME, Yakovleva TV, Murray JS, Siahaan TJ. Inhibition of LFA-1/ICAM-1 and VLA-4/VCAM-1 as a therapeutic approach to inflammation and autoimmune diseases. Med Res Rev. 2002;22(2):146-167.

16. Delahaye NF, Coltel N, Puthier D, Barbier M, Benech P, Joly F, et al. Gene expression analysis reveals early changes in several molecular pathways in cerebral malaria-susceptible mice versus cerebral malaria-resistant mice. BMC Genomics. 2007;8:452.

17. Morrell CN. Understanding platelets in malaria infection. Curr Opin Hematol. 2014;21(5):445-449. doi: 10.1097/ MOH.0000000000000073.

18.Vaughan AM, Kappe SH, Ploss A, Mikolajczak SA. Development of humanized mouse models to study human malaria parasite infection. Future Microbiol. 2012; 7(5): 657-665. doi: 10.2217/fmb.12.27.

19. Srivastava K, Field DJ, Aggrey A, Yamakuchi M, Morrell CN. Platelet Factor 4 Regulation of Monocyte KLF4 in Experimental Cerebral Malaria. PLoS One. 2010;5(5):e10413. doi: 10.1371/journal. pone.0010413.

20. Aggrey AA, Srivastava K, Ture S, Field DJ, Morrell CN. Platelet induction of the acute-phase response is protective in murine experimental cerebral malaria. J Immunol. 2013;190(9):4685-4691. doi: 10.4049/ jimmunol.1202672.

21. Chapman LM, Aggrey AA, Field DJ, Srivastava K, Ture S, Yui K, et al. Platelets present antigen in the context of MHC class I. J Immunol. 2012;189(2):916-923. doi: 10.4049/jimmunol.1200580. Epub 2012 Jun 15.

22. Hafalla JC, Cockburn IA, Zavala F. Protective and pathogenic roles of CD8+ T cells during malaria infection. Parasite Immunol. 2006;28(12):15-24. 
23. Morrell CN, Srivastava K, Swaim A, Teresa M, Nagineni C, Hooks JJ, et al. Interferon- $\beta$ Suppresses the Development of Experimental Cerebral Malaria. Infect. Immun. doi:10.1128/IAI.00810-10.

24. McMorran BJ, Wieczorski L, Drysdale KE, Chan JA, Huang HM, Smith C, et al. Platelet factor 4 and Duffy antigen required for platelet killing of Plasmodium falciparum. Science. 2012;338(6112):1348-1351. doi: 10.1126/science.1228892.

25. Nesmelova IV, Sham Y, Dudek AZ, van Eijk LI, Wu G, Slungaard A, et al. Platelet factor 4 and interleukin-8 CXC chemokine heterodimer formation modulates function at the quaternary structural level. J Biol Chem. 2005;280(6):4948-4958.

26. Iida Y, Xu B, Xuan H, Glover KJ, Tanaka H, Hu X, et al. Peptide inhibitor of CXCL4-CCL5 heterodimer formation, MKEY, inhibits experimental aortic aneurysm initiation and progression. Arterioscler Thromb Vasc Biol. 2013;33(4):718-726. doi: 10.1161/ATVBAHA.112.300329.

27. Gleissner CA, Hundelshausen PV, Ley K: Platelet Chemokines in Vascular Disease. Arterioscler Thromb Vasc Biol. 2008;28(11):19201927. doi: 10.1161/ATVBAHA.108.169417.

28. Weber C. Platelets and chemokines in atherosclerosis: partners in crime. Circ Res. 2005;96(6):612-6.

29. Nayak KC, Mohini, Kumar S, Tanwar RS, Kulkarni V, Gupta A, et al A study on pulmonary manifestations in patients with malaria from northwestern India (Bikaner). J Vector Borne Dis. 2011;48(4):219223.
30.Zarbock A, Ley K. The role of platelets in acute lung injury (ALI). Front Biosci (Landmark Ed). 2009;14: 150-158.

31. Srivastava K: The Role of Platelets in Malarial Acute Lung Injury and Acute Respiratory Distress Syndrome: A World of Possibilities. Acta Medica International; 2014;1(2):117-123.

32. Mock JR, Garibaldi BT, Aggarwal NR, Jenkins J, Limjunyawong $\mathrm{N}$, Singer BD, et al. Foxp3+ regulatory $\mathrm{T}$ cells promote lung epithelial proliferation. Mucosal Immunol. 2014;7(6):1440-1451. doi: 10.1038/ mi.2014.33.

33. Garibaldi BT, D’Alessio FR, Mock JR, Files DC, Chau E, Eto Y, et al. Regulatory $\mathrm{T}$ Cells Reduce Acute Lung Injury Fibroproliferation by Decreasing Fibrocyte Recruitment. Am J Respir Cell Mol Biol. 2013;48(1):35-43. doi: 10.1165/rcmb.2012-01980C.

34. Pain A, Ferguson DJP, Kai O, Urban BC, Lowe B, Marsh K, et al. Plateletmediated clumping of Plasmodium falciparum-infected erythrocytes is a common adhesive phenotype and is associated with severe malaria. Proc Natl Acad Sci U S A. 2001;98(4):1805-1810.

35. Thakur RS, Tousif S, Awasthi V, Sanyal A, Atul PK, Punia P, et al. Mesenchymal stem cells play an important role in host protective immune responses against malaria by modulating regulatory $\mathrm{T}$ cells. Eur J Immunol. 2013;43(8):2070-2077. doi: 10.1002/eji.201242882. 